\title{
Acoustic tweezing cytometry enhances osteogenesis of human mesenchymal stem cells through cytoskeletal contractility and YAP activation
}

\author{
Xufeng Xue ${ }^{1}$, Xiaowei Hong ${ }^{2}$, Zida $\mathrm{Li}^{1}$, Cheri X. Deng ${ }^{1,2, *}$ and Jianping $\mathrm{Fu}^{1,2,3, *}$ \\ ${ }^{1}$ Department of Mechanical Engineering, University of Michigan, Ann Arbor, MI 48109, USA; \\ ${ }^{2}$ Department of Biomedical Engineering, University of Michigan, Ann Arbor, MI, 48109, USA; \\ ${ }^{3}$ Department of Cell and Developmental Biology, University of Michigan Medical School, Ann \\ Arbor, MI 48109, USA.
}

*Correspondence should be addressed to J.F. (jpfu@umich.edu) or C.X.D. (cxdeng @umich.edu) 


\begin{abstract}
Human mesenchymal stem cells (hMSCs) have great potential for cell-based therapies for treating degenerative bone diseases. It is known that mechanical cues in the cell microenvironment play an important role in regulating osteogenic (bone) differentiation of hMSCs. However, mechanoregulation of lineage commitment of hMSCs in conventional twodimensional (2D) monocultures or bioengineered three-dimensional (3D) tissue constructs remains suboptimal due to complex biomaterial design criteria for hMSC culture. In this study, we demonstrate the feasibility of a novel cell mechanics and mechanobiology tool, acoustic tweezing cytometry (ATC), for mechanical stimulation of hMSCs. ATC utilizes ultrasound (US) pulses to actuate functionalized lipid microbubbles (MBs) which are covalently attached to hMSCs via integrin binding to exert forces to the cells. ATC stimulation increases cytoskeletal contractility of hMSCs regardless of the cell area. Furthermore, ATC application rescues osteogenic differentiation of hMSCs in culture conditions that are intrinsically repressive for hMSC osteogenesis (e.g., soft cell culture surfaces). ATC application activates transcriptional regulator YAP to enhance hMSC osteogenesis. Our data further show that F-actin, myosin II, and RhoA/ROCK signaling function upstream of YAP activity in mediating ATC-stimulated hMSC osteogenesis. With the capability of applying controlled dynamic mechanical stimuli to cells, ATC provides a powerful tool for mechanoregulation of stem cell behaviors in tissue engineering and regenerative medicine applications.
\end{abstract}

\title{
KEY WORDS
}

Human mesenchymal stem cells; Osteogenesis; Acoustic tweezing cytometry; Mechanotransduction; YAP signaling 


\section{INTRODUCTION}

Human mesenchymal stem cells (hMSCs), which can be readily isolated from adult tissues such as bone marrow and adipose tissues, have great potential in tissue engineering and regenerative medicine owing to their multipotency and good accessibility [1]. In particular, osteogenic differentiation of hMSCs in conjunction with tissue engineering methods have been exploited to provide an alternative approach besides autologous bone graft to replace or restore damaged bone tissues [2]. For example, biomimetic constructs composed hydroxyapatite (HA) and loaded with hMSCs have been shown to significantly enhance bone formation compared with HA alone at 12 weeks after surgery in vivo [3]. Cellular inflammatory response was less severe with hMSC loaded HA constructs compared with HA alone. This clearly shows hMSCs as a promising cell source for use in bone tissue engineering, given their regenerative and immunomodulatory properties. A major challenge, however, is to efficiently guide osteogenic differentiation of hMSCs either in conventional two-dimensional (2D) monocultures or bioengineered threedimensional (3D) tissue constructs [4]. Mechanical cues such as extracellular matrix (ECM) stiffness, cell shape, and mechanical forces have all been shown to play significant roles in regulating lineage commitment and differentiation of hMSCs [5-12]. Specifically, it is known that hMSCs tend to exhibit limited osteogenic potential when cultured in a 3D environment that is mechanically soft (e.g., a soft hydrogel) or provides confined spaces to the cells [7, 13]. Given the significant interest in applying hMSCs osteogenesis in conjunction with suitable 2D or 3D culture systems for bone tissue engineering, it is important to develop techniques that can efficiently promote osteogenic differentiation of hMSCs in different culture environments especially those that are intrinsically suboptimal for hMSC osteogenesis. 
Recent studies have shown that the RhoA/ROCK/myosin-II signaling axis, which is the major biochemical pathway mediating the cytoskeletal contractility in non-muscle mammalian cells, plays a critical role in regulating hMSC osteogenesis [5, 14, 15]. Importantly, RhoA/ROCK-mediated cytoskeletal contractility is critically involved in mechanotransduction in hMSCs [16-19]. Recent studies also indicate the importance of YAP signaling in controlling mechanosensitive hMSC osteogenesis, highlighting mechanotransductive mechanisms acting upstream of YAP transcriptional activity through Rho GTPases, cytoskeletal contractility, and Factin mechanics [8, 9, 20-24]. Altogether, mechanosensitive hMSC osteogenesis has been suggested to be regulated through RhoA/ROCK/myosin-II signaling axis and the mechanosensitive transcriptional regulator YAP; however, the functional link between the RhoA/ROCK/myosin-II signaling axis and YAP activity in hMSCs remains to be elucidated.

We have recently developed a novel method, acoustic tweezing cytometry (ATC), for applying controllable subcellular forces to a large number of cells simultaneously [25, 26] (Fig. 1A). In ATC, RGD-peptide-functionalized lipid microbubbles (MBs) are first attached to live cells via RGD-integrin binding. Ultrasound (US) pulses are then applied to generate volume pulsation and translational movement of the integrin-anchored MBs, thereby exerting external forces in $\mathrm{pN}$ - $\mathrm{nN}$ range to the cells at the location of $\mathrm{MB}$ attachment. We have previously shown that ATC application can elicit a rapid and sustained increase in the cytoskeletal contractility of adherent mechanosensitive cells, including hMSCs [25, 26], demonstrating the potential of applying ATC for biomechanical stimulation of adherent mechanosensitive cells using MBs as an actuatable and biocompatible agent [25-27].

In this work, we employed ATC for mechanical stimulation of hMSCs to guide their osteogenic differentiation. We show that ATC application increases cytoskeletal contractility of 
hMSCs independent of the cell area. Importantly, ATC stimulation rescues osteogenic differentiation of hMSCs cultured on soft substrates that are intrinsically prohibitive for hMSC osteogenesis. Our data further show that in ATC-mediated mechanotransduction in hMSCs, YAP functions as a downstream nuclear transducer and effector of cytoskeletal contractile force to mediate mechanosensitive hMSC osteogenesis.

\section{METHODS}

\section{Fabrication and surface functionalization of PDMS micropost array}

The PDMS micropost array (PMA) was fabricated using a protocol described previously [28]. Briefly, the PMA was generated using replica molding from a silicon mold fabricated by standard photolithography and deep reactive ion etching (DRIE). The center-to-center spacing between adjacent hexagonally spaced microposts in the PMA was $4 \mu \mathrm{m}$. The micropost diameter $D$ was $1.83 \mu \mathrm{m}$, and the micropost height $L$ was modulated by controlling DRIE etching time from $0.7 \mu \mathrm{m}$ (for rigid PMA substrate) to $14.5 \mu \mathrm{m}$ (for soft PMA substrate). The PMA with a post height $L$ of $7.1 \mu \mathrm{m}$ was used for intracellular contractile force measurements. The spring constant $K$ of micropost was calculated using the Euler-Bernoulli beam theory as $K=3 \pi E D^{4}$ / $\left(64 L^{3}\right)$, where $E$ is the Young's modulus of PDMS. The effective substrate rigidity $E_{\text {eff }}$ of the PMA was then calculated as $E_{\text {eff }}=9 K /(2 \pi D)$ [29]. In cellular contractile force measurements, a coverslip holding the PMA was attached to the bottom of a glass-bottom 6-well plate (MatTek). In all other experiments, coverslips holding the PMA were placed into standard 12-well tissue culture plates. To promote cell adhesion, the PMA top surface was functionalized with fibronectin (Sigma-Aldrich) using microcontact printing as described previously [28]. To confine

cell areas on the PMA, PDMS stamps with $30 \times 30 \mu \mathrm{m}^{2}, 50 \times 50 \mu \mathrm{m}^{2}, 100 \times 100 \mu \mathrm{m}^{2}$ square 
patterns were used for microcontact printing. In cellular contractile force measurements, the PMA was further labeled with $5 \mu \mathrm{g} \mathrm{mL}^{-1}$ DiI (Invitrogen) for $1 \mathrm{hr}$ before blocking using pluronics (Sigma-Aldrich) as described previously [28].

\section{Cell culture}

Human mesenchymal stem cells (hMSCs; Lonza) were maintained in human mesenchymal stem cell growth medium (Lonza) at $37^{\circ} \mathrm{C}$ and $5 \% \mathrm{CO}_{2}$. For osteogenic differentiation, culture medium was switched to osteogenic differentiation medium (Lonza) $1 \mathrm{~d}$ after hMSCs were seeded. For all experiments, hMSCs at passages $3-6$ were plated at a density of 3,000 cells cm$^{-2}$, and osteogenic differentiation medium was changed every other day. hMSCs were authenticated by Lonza using immunostaining, characteristic cell morphology, and flow cytometry. Cells were tested for mycoplasma contamination using the LookOut mycoplasma detection kit (SigmaAldrich).

\section{Attachment of targeted lipid microbubbles to cells}

Targesphere $^{\mathrm{TM}}$-SA lipid microbubbles $(\mathrm{MBs})$ (mean diameter of $2.3 \pm 0.2 \mu \mathrm{m}$; Targeson) were mixed with biotinylated Arg-Gly-Asp (RGD) peptides (Peptides International) with a volumetric ratio of 5:1 between $\mathrm{MBs}\left(3 \times 10^{9} \mathrm{~mL}^{-1}\right)$ and $\mathrm{RGD}\left(2 \mathrm{mg} \mathrm{mL}^{-1}\right)$ for $30 \mathrm{~min}$ at room temperature to allow functionalization of MBs with RGD. To attach RGD-coated MBs (RGD-MBs) to cells, culture medium was first removed from 12-well tissue culture plates before $50 \mu \mathrm{L}$ diluted RGDMB solution (1:75 dilution in culture medium) was added to each well of the 12-well plate. The tissue culture plate was flipped upside down for $10 \mathrm{~min}$ to facilitate attachment of RGD-MBs to 
cell membrane via RGD-integrin binding. Unbound MBs were removed from cell culture by gently washing with culture medium.

\section{Ultrasound stimulation}

A $1 \mathrm{MHz}$ planar ultrasound (US) transducer (Olympus; $3 \mathrm{~dB}$, beam width of $12 \mathrm{~mm}$ and a Rayleigh distance of $54 \mathrm{~mm}$ ) submerged in a water tank was used for ATC application. The US transducer was positioned at an angle of $45^{\circ}$ relative to the vertical direction facing upward at the Rayleigh distance from cells at the bottom of tissue culture plates. Tissue culture plates were positioned with only their bottoms submerged in water to allow acoustic coupling. The

temperature of water in the tank was kept at $37{ }^{\circ} \mathrm{C}$. The US transducer was driven by a waveform generator (Agilent Technologies) and a $75 \mathrm{~W}$ power amplifier (Amplifier Research) to generate US pulses with $10 \mathrm{~Hz}$ pulse repetition frequency (PRF) and 50\% duty cycle. The US transducer was calibrated in free field in water using a needle hydrophone (HNR-0500, Onda). US pulses with an acoustic pressure amplitude of 0.025 or $0.08 \mathrm{MPa}$ were used in this study.

\section{Quantification of cellular contractile force using PDMS micropost array}

Fluorescence microscopy images showing the PMA top surface were obtained using an inverted epi-fluorescence microscope enclosed in an incubator maintaining cell culture at $37^{\circ} \mathrm{C}$ and $5 \%$ $\mathrm{CO}_{2}$ (Carl Zeiss MicroImaging). Images were analyzed using a custom-developed MATLAB program to quantify micropost deflections from their original, unbent positions [28]. Horizontal cellular contractile forces were obtained by multiplying micropost deflections with the micropost spring constant $K$. 


\section{Immunostaining}

Cells were fixed with $4 \%$ paraformaldehyde (Electron Microscopy Sciences) at room temperature for $30 \mathrm{~min}$ and permeabilized with $0.2 \%$ Triton X-100 (Sigma-Aldrich) for another 30 min. After blocking using 10\% goat serum (Thermo Fisher Scientific) for $1 \mathrm{hr}$ at room temperature, cells were incubated with the primary antibody against YAP (Cell Signaling Technologies) diluted in $10 \%$ goat serum for $1 \mathrm{hr}$. Cells were then incubated with a secondary antibody (Santa Cruz) for $1 \mathrm{hr}$ at room temperature. Cell nucleus was stained with 4,6-diamino2-phenylindole (DAPI; Invitrogen), while actin filaments were stained with Alexa Fluor 555 conjugated phalloidin (Thermo Fisher Scientific). Cell areas were quantified with ImageJ using fluorescence images of F-actin. Percentage of cells with nuclear YAP was calculated as the ratio of the number of hMSCs in which YAP fluorescence intensity was greater in nucleus than in cytosol and the total cell number.

To quantify hMSC osteogenesis, cells were stained for alkaline phosphatase (ALP) using the alkaline phosphatase kit (Sigma-Aldrich) per manufacturer's instructions. Briefly, cells were fixed with $4 \%$ paraformaldehyde before stained with fast red violet LB/naphthol (Sigma-Aldrich) to visualize ALP. Cell nucleus was counterstained using hematoxylin. Percentage of hMSC osteogenesis was calculated as the ratio of ALP-positive cell number and the total cell number.

\section{Real-time PCR analysis}

Total RNA was isolated using RNAeasy kit (Qiagen) and reverse transcribed to cDNA with iScript cDNA synthesis kit (Bio-rad). RT-PCR was performed using the CFX connect real-time PCR detection system (Bio-rad) with SYBR Green PCR mastermix. TBP was used as an endogenous control for relative quantifications. Primers used in this work include: Osteocalcin 
(forward: 5'-CCACCGAGACACCATGAGAG-3', and reverse: 5'-

TGCTTGGACACAAAGGCTGC-3'), Osteopontin (forward: 5'-

AAACGCCGACCAAGGAAAAC-3', and reverse: 5'-GCTTCTGAGATGGGTCAGGG-3'), and BSP (forward: 5'-GCCAGAGGAAGCAATCACCA-3', and reverse: 5'TGCCCTGAACTGGAAATCGT-3’).

\section{Western blot}

Whole-cell lysates were prepared from hMSCs using Laemmli Sample Buffer (Bio-Rad) and homogenized by sonication. Proteins in lysates were separated using SDS-polyacrylamide gel electrophoresis before transferred to PVDF membranes. After blocking with a blocking buffer (Li-Cor) for $1 \mathrm{hr}$, the PVDF membrane was incubated with the primary antibody against YAP (Cell Signaling Technologies) at $4^{\circ} \mathrm{C}$ overnight. The membrane was then incubated with a secondary antibody (Li-Cor) for $1 \mathrm{hr}$ before imaging with Li-Cor Odyssey Sa Infrared Imaging system (Li-Cor).

\section{Statistical analysis}

All experiments were conducted in $n>3$ biological replicates and repeated in $n>3$ independent experiments. Data with error bars are presented as the mean \pm SEM. Statistical analysis was conducted using independent, two-tailed Student's $t$-test in Excel (Microsoft). For all comparisons, $P<0.05$ was considered statistically significant.

\section{RESULTS AND DISCUSSION}




\section{ATC increases cytoskeletal contractility of hMSCs independent of cell area}

To explore the feasibility of using ATC to enhance hMSC osteogenesis, we first examined the effect of ATC on intracellular cytoskeletal contractility of hMSCs. We utilized an elastomeric micropost array made with silicone elastomer polydimethylsiloxane (PDMS), or PDMS micropost array (PMA), which have been developed as subcellular live-cell force sensors to quantify cytoskeletal contractile forces of adherent mammalian cells [13, 28, 30]. The PMA consists of a regular array of uniformly spaced, vertical, elastomeric posts fabricated using photolithography and replica molding. The post tips are coated with adhesive proteins using microcontact printing. Cells are able to adhere, spread out, and exert contractile forces that deflect underlying posts. Each post, therefore, functions as a cantilever, capable of measuring local cellular contractile force at the post tip. For tip deflections $\delta$ that are small compared to the post height $L$, the PDMS post can be modeled as a linearly elastic beam subjected to pure bending. Thus, intracellular contractile force $F$ exerted at the tip of the PDMS post can be calculated as $F=K \delta$, with the post spring constant $K=3 \pi E D^{4} /\left(64 L^{3}\right)$, where $E$ is the elastic modulus of PDMS and $D$ is the post diameter.

In this study, to track long-term cytoskeletal contractile force dynamics of individual hMSCs in response to ATC application in a controlled environment, we first patterned single hMSCs on isolated, square-shaped adhesive islands $\left(75 \times 75 \mu \mathrm{m}^{2}\right)$ on the PMA (Fig. 1B). Patterning of the cells not only restricted direct cell-cell interactions and homogenized cell shape, but also permitted tracking of individual hMSCs without the added difficulty of accounting for cell migration. MBs (mean diameter of $2.3 \mu \mathrm{m}$ ) functionalized with Arg-Gly-Asp (RGD) peptides were then attached to hMSCs through specific binding to integrin on the cell membrane 
(See Method). The average number of MBs attached to each cell was $6.97 \pm 3.09$ / cell (mean \pm standard deviation) (Fig. 1A-C).

After individual hMSCs were loaded with MBs, ultrasound (US) pulses were delivered at an acoustic pressure of $0.08 \mathrm{MPa}$, with center frequency of $1 \mathrm{MHz}$, pulse repetition frequency (PRF) of $10 \mathrm{~Hz}$, and pulse duration of $50 \mathrm{~ms}$ to stimulate individual patterned hMSCs for a total period of $30 \mathrm{~s}$ (See Method). Under the influence of US pulses, MBs underwent volume pulsation at the US frequency of $1 \mathrm{MHz}$, generating fluid microstreaming surrounding MBs that exert a local shear force to MB-loaded hMSCs [26]. More notably, during each US pulse (duration of $50 \mathrm{~ms}$ ), the US field generated a directional acoustic radiation force of $30.62 \mathrm{nN}$ on an individual $\mathrm{MB}$, displacing the $\mathrm{MB}$ without detaching it from the cells, thereby generating local forces to MB-loaded hMSCs by straining the RGD-integrin-cytoskeleton connection [25, 26]. Between US pulses, the MBs retracted back toward their original positions on the cell membrane when there was no acoustic radiation force acting on the MBs. After ATC application, cytoskeletal contractile force of patterned single hMSCs increased rapidly, with subcellular responses concentrating at the cell periphery (Fig. 1D). Specifically, 30 min after ATC stimulation, the total cytoskeletal contractility of patterned single hMSCs (+US +MB) increased to $115.8 \% \pm 2.7 \%$ (mean \pm standard error of mean; $n=6$ ) compared to the pre-ATC contractile level. This elevated contractile state of patterned single hMSCs maintained for about $1 \mathrm{hr}$ before gradually reversing back to the pre-ATC level at $5 \mathrm{hr}$ (Fig. 1D). In comparison, patterned single hMSCs in control groups without targeted MBs and without US application (-US -MB) exhibited no observable change in their cytoskeletal contractile force (Fig. 1D). These results support that as a regulated cellular homeostatic property $[29,31,32]$, whole-cell cytoskeletal contractility of hMSCs can be activated by subcellular force stimulation from ATC application. 
Our previous study has suggested that cytoskeletal contractile response of adherent mammalian cells to ATC application depends on US parameters [25]. Thus, we measured contractile responses of patterned hMSCs to ATC stimulation using a lower acoustic pressure of 0.025 MPa while keeping the other US parameters unchanged. This lower acoustic pressure amplitude corresponds to a lower acoustic radiation force of $2.99 \mathrm{nN}$ on a single $\mathrm{MB}$ [25]. With an acoustic pressure of $0.025 \mathrm{MPa}, \mathrm{MB}$ translational movements were much smaller than those observed under an acoustic pressure of $0.08 \mathrm{MPa}$. Correspondingly, the total cytoskeletal contractility of patterned single hMSCs at 30 min after ATC application exhibited a limited increase, up to $107.5 \% \pm 4.2 \%(n=7)$ of the pre-US contractile level. This relatively limited increase of total cytoskeletal contractility quickly returned to the pre-ATC contractile level at 1 $\mathrm{hr}$ and remained so for the next $4 \mathrm{hr}$ (Fig. 1E). These results suggest that the capability of ATC to increase cytoskeletal contractility of hMSCs depends on the acoustic radiation force or acoustic pressure amplitude of applied US pulses. ATC stimulation with US pulses with an acoustic pressure of $0.08 \mathrm{MPa}$ could significantly increase the cytoskeletal contractility of patterned single hMSCs.

We next examined whether the impact of ATC stimulation on cytoskeletal contractile force of patterned single hMSCs depends on the initial cellular contractile state or cell spread area. In these experiments, single hMSCs were patterned on isolated, square-shaped adhesive islands of different areas $\left(50 \times 50 \mu \mathrm{m}^{2}, 75 \times 75 \mu \mathrm{m}^{2}\right.$, and $\left.100 \times 100 \mu \mathrm{m}^{2}\right)$ on the PMA (Fig. 2A). Consistent with previous reports $[16,18,30]$, without ATC stimulation, the total cytoskeletal contractile force of patterned single hMSCs was positively correlated with cell spread area, supporting the established notion that cell spread area is an important regulator of the formation and integrity of the actin cytoskeleton $[16,18,30]$ (Fig. 2B). Interestingly, patterned single 
hMSCs with different cell areas showed comparable levels of contractile force increase in response to ATC application (acoustic pressure $0.08 \mathrm{MPa}$, center frequency $1 \mathrm{MHz}, \mathrm{PRF} 10 \mathrm{~Hz}$, pulse duration $50 \mathrm{~ms}$, and total duration $30 \mathrm{~s}$ ) (Fig. 2C). Regardless of cell area, total contractile forces of patterned single hMSCs (measured at 30 min after ATC application) all increased 15\% - $18 \%$ relative to pre-US levels, i.e. $118.2 \% \pm 1.7 \%$ for $50 \times 50 \mu \mathrm{m}^{2}(n=9), 115.8 \% \pm 2.7 \%$ for $75 \times 75 \mu \mathrm{m}^{2}(n=6)$, and $116.3 \% \pm 1.5 \%$ for $100 \times 100 \mu \mathrm{m}^{2}(n=7)$. Contractile forces of patterned single hMSCs gradually decreased back to the pre-US contractile levels at $5 \mathrm{hr}$. As expected, patterned single hMSCs with different cell areas in control groups without MBs and without US treatments (-US -MB) exhibited no observable change in their cytoskeletal contractile force (Fig. 2D). Together, these results suggest that ATC-induced increase of cytoskeletal contractility in hMSCs is independent of cell area or the contractile state before ATC application.

\section{ATC rescues hMSC osteogenesis on soft substrates without affecting cell spread area}

Previous studies have shown the importance of early cytoskeletal contractile responses in the hMSC differentiation process, and in particular, it has been demonstrated that elevated cytoskeletal contractile states of single hMSCs strongly correlate with their later osteogenic commitment [13]. Therefore, our results of ATC-induced increase in cytoskeletal contractility regardless of the cell area of hMSCs (Fig. 2) suggest an interesting possibility that ATC could stimulate cytoskeletal contractility and thus rescue hMSC osteogenesis on soft substrates or with confined cell areas.

To modulate substrate rigidity, here we used the PMA system with tunable rigidities, achieved by controlling PDMS micropost heights while preserving the same surface geometry 
(i.e., micropost diameter and center-to-center distance) to effectively decouple substrate rigidity from adhesive and surface properties [13, 28]. Micropost height determines the degree to which a post bends in response to a horizontal cellular contractile force. Thus, a PMA with longer posts provides a softer substrate for culturing hMSCs, whereas a PMA with shorter posts provides a more rigid substrate. For this study, we chose two PMAs with the effective substrate rigidity $E_{\text {eff }}$ $=1 \mathrm{KPa}$ (soft PMA substrate) and $E_{\text {eff }}=1.2 \mathrm{MPa}$ (rigid PMA substrate) to compare the response of hMSCs to ATC stimulation.

Osteogenic differentiation of hMSCs was assessed by examining alkaline phosphatase (ALP) activity, a marker of hMSC osteogenesis. As expected, after culturing in osteogenic differentiation medium for 6 days, hMSCs without MBs and ATC stimulation (-US -MB) exhibited greater osteogenesis on the rigid PMA than on the soft PMA, as evidenced by distinct numbers of $\mathrm{ALP}^{+}$cells on the corresponding PMA substrates (Fig. 3A\&3B). For hMSCs seeded on the rigid PMA, with or without targeted MBs, US application (5 min each day for 6 days; US parameters: center frequency $1 \mathrm{MHz}$, pulse repetition frequency $10 \mathrm{~Hz}$, pulse duration $50 \mathrm{~ms}$, acoustic pressure $0.08 \mathrm{MPa}$ ) did not significantly enhance hMSC osteogenesis compared with untreated control (-US -MB; $n=3$ ) (Fig. 3A\&B). This could be due to the fact that the percentage of hMSCs that had committed to osteogenesis on the right PMA might have reached a maximum level. It remains to be determined whether increasing the dose (US stimulation time) of ATC stimulation could further enhance osteogenesis of hMSCs cultured on the rigid PMA. Interestingly, on the soft PMA, for hMSCs loaded with targeted MBs and subjected to ATC application (+US +MB) (5 min each day for 6 days; US parameters: center frequency $1 \mathrm{MHz}$, pulse repetition frequency $10 \mathrm{~Hz}$, pulse duration $50 \mathrm{~ms}$, acoustic pressure $0.08 \mathrm{MPa}$ ), osteogenesis, as indicated by the percentage of $\mathrm{ALP}^{+}$cells, reached $36.1 \% \pm 1.5 \%$. This is 
comparable to the percentage of $\mathrm{ALP}^{+}$cultured on the rigid PMA and is significantly higher than the two control groups $(21.2 \% \pm 0.3 \%$ for $-\mathrm{US}-\mathrm{MB}$ and $20.58 \% \pm 1.98 \%$ for $+\mathrm{US}-\mathrm{MB})$ (Fig. 3A\&B). When hMSCs were stimulated by ATC for only $30 \mathrm{~s}$ while keeping other US parameters unchanged, percentage of ALP+ hMSCs cultured on the soft PMA didn't show significant difference compared with untreated control (data not shown).

To further assess osteogenic differentiation of hMSCs subjected to ATC treatments, we analyzed gene expression of three different osteogenic markers, osteocalcin (OCN), osteopontin (OPN), and bone sialoprotein (BSP) using qRT-PCR. Consistent with ALP activity assays, for hMSCs without MBs and ATC stimulation (-US -MB), expression levels of these three genes were significantly higher for hMSCs on the rigid PMA compared with on the soft PMA (OCN: 2.8-fold higher and $P=0.049$; OPN: 2.3 -fold higher and $P=0.041$; BSP: 2.8 -fold higher and $P=$ 0.046) (Fig. 3C). Interestingly, for hMSCs seeded on the soft PMA, ATC application significantly increased expression of OCN, OPN, and BSP for hMSCs with MBs and subjected to US excitation (+US +MB) when compared with hMSCs without MBs and ATC stimulation (US -MB) (Fig. 3C). Specifically, for hMSCs cultured on the soft PMA and loaded with MBs, ATC treatment led to 7.0-fold increase for OCN $(P=0.047), 2.0$-fold increase for OPN $(P=$ 0.021), and 5.4-fold increase for BSP $(P=0.042)$, respectively. In contrast, for hMSCs seeded on the rigid PMA, regardless whether or not hMSCs were loaded with MBs, US stimulation did not significantly increase OCN, OPN, or BSP expression compared with the control group (-US -MB; Fig. 3C). Together, these results show that ATC rescues hMSC osteogenesis on soft substrates that are intrinsically repressive for osteogenesis, possibly through increased cytoskeletal contractility responding to subcellular force stimulations from ATC application. 
Since cell spread area can act as an independent regulator of hMSC osteogenesis, with large cell spread area promoting hMSC osteogenesis $[5,6,13]$, we next specifically examined whether ATC stimulation led to a change of cell spread area, thereby modulating hMSC osteogenesis. To this end, ATC stimulation was applied to hMSCs loaded with MBs and seeded on either the rigid or soft PMA (US conditions: acoustic pressure $0.08 \mathrm{MPa}$, center frequency 1 MHz, PRF $10 \mathrm{~Hz}$, pulse duration $50 \mathrm{~ms}$, and total US stimulation duration $5 \mathrm{~min}$ ). Cells were fixed 30 min after ATC treatment and stained for F-actin to visualize cell spread areas (Fig. 4A). As expected, for hMSCs without MBs and US application (-US -MB), cell spread area for the rigid PMA group, $6626.12 \pm 286.29 \mu \mathrm{m}^{2}(n=182)$, was much greater than the cell area for the soft PMA group, $1430.46 \pm 65.05 \mu \mathrm{m}^{2}(n=161)$ (Fig. 4B). Interestingly, ATC treatment did not result in significant changes of cell spread areas for hMSCs plated on either the rigid or soft PMA. hMSCs loaded with MBs and subjected to ATC treatments exhibited very similar cell spread areas as those without ATC stimulation (Fig. 4B).

\section{ATC upregulated YAP activity}

YAP is a mechanosensitive transcriptional regulator that responds to mechanical cues such as extracellular matrix rigidity, cell spread area, and cytoskeletal contractility in both adult somatic cells and embryonic stem cells by dynamically shuttling in and out of the nucleus [23, 33, 34]. Active YAP localizes in the nucleus of mechanosensitive adherent cells seeded on rigid substrates, whereas YAP moves out of the nucleus and remains in the cytoplasm for cells cultured on soft substrates. In parallel, mechanosensitive adherent cells cultured on large micropatterned adhesive islands exhibit greater cytoskeletal contractile force and nuclear localization of YAP, whereas cells confined to small adhesive islands show limited cytoskeletal 
contractile force and inactive YAP retained in the cytoplasm. Importantly, YAP is directly involved in the transcriptional program mediating force-sensitive hMSC osteogenesis $[8,9,23$, 24, 33]. hMSCs with nuclear localized YAP are prone to osteogenic differentiation [8, 23, 24, 33].

To elucidate the role of YAP in ATC-stimulated osteogenic differentiation of hMSCs, we conducted experiments where transcriptional activity of YAP was inhibited by supplementing the osteogenic differentiation medium with verteporfin $(5 \mu \mathrm{M})$ on day 1 and day 2 . Verteporfin, independent of its photosensitive property, inhibits the interaction of YAP with TEAD by upregulating 14-3-36, a scaffold protein that binds and retains YAP in cytoplasm and targets it for degradation [35]. Verteporfin is photosensitive, and when stimulated by red light at $689 \mathrm{~nm}$, verteporfin could produce reactive oxygen radicals. However, in our experiments, the effect of verteporfin on YAP inhibition is not photoinduced, and hMSCs treated with verteporfin were kept in incubator without light exposure for most of time. Thus, verteporfin used in our assays should not induce off-target effects associated with its photosensitive property. Western blot data showed that verteporfin treatment suppressed YAP expression in hMSCs (Fig. 5A). Importantly, hMSCs treated with verteporfin showed significant lower expression of all three osteogenic markers than control (2.6-fold decrease for OCN, with $P=0.027$; 2.2 -fold decrease for OPN, with $P=0.016$; 4.5 -fold decrease for BSP, with $P=0.031$; Fig. 5B) after $6 \mathrm{~d}$ of culture in the osteogenic differentiation medium on tissue culture plates, supporting the involvement of YAP in the transcriptional program driving hMSC osteogenesis.

Since YAP activity is sensitive to mechanical cues in the local cell microenvironment such as extracellular matrix stiffness, cell shape, and cytoskeletal contractility, we sought to examine the response of YAP activity in hMSCs subjected to ATC stimulation. To this end, 
expression and subcellular localization of YAP were examined for hMSCs cultured on both the soft and rigid PMAs 30 min after ATC stimulation (US conditions: acoustic pressure $0.08 \mathrm{MPa}$, center frequency $1 \mathrm{MHz}, \mathrm{PRF} 10 \mathrm{~Hz}$, pulse duration $50 \mathrm{~ms}$, and total US stimulation duration 5 min). As expected, for hMSCs without MBs and ATC stimulation (-US -MB), the percentage of hMSCs with nuclear YAP was significant higher for the rigid PMA $(91.07 \% \pm 2.17 \% ; n=3)$ than the soft PMA $(15.81 \% \pm 2.37 \% ; n=3)$ (Fig. 6A\&B). Importantly, ATC stimulation significantly increased the percentage of hMSCs with nuclear YAP for the cells loaded with MBs and cultured on the soft PMA $(41.7 \% \pm 3.53 \% ; n=3)$ (Fig. 6A\&B). Similar to its effect on hMSC osteogenesis on the rigid PMA, ATC stimulation did not significantly affect YAP nuclear localization for hMSCs on the rigid PMA, regardless whether or not the cells were pre-coated with MBs, likely due to the fact that even without ATC treatments, almost all hMSCs on the rigid PMA already exhibited nuclear YAP (Fig. 6B). We should note that there was a slight but not significant increase of nuclear YAP for hMSCs on the soft PMA by US exposure alone without MBs (+US -MB; Fig. 6B). Together, these data support that US stimulation alone does not have a significant effect on YAP activity in hMSCs, and it is through mechanical stimulations via the MB-integrin linkage that transmits the acoustic radiation force to the cells leading to mechanoresponsive YAP activity in hMSCs.

Cell spread area can also regulate subcellular localization of YAP, with small cell spread area promoting YAP cytoplasmic localization [20, 23, 33]. Thus, we further examined whether ATC stimulation could promote nuclear localization of YAP in patterned single hMSCs with defined cell areas of different sizes $\left(30 \times 30 \mu \mathrm{m}^{2}\right.$ and $\left.100 \times 100 \mu \mathrm{m}^{2}\right)$. hMSCs were stained for YAP 30 min after ATC application (US conditions: acoustic pressure $0.08 \mathrm{MPa}$, center frequency $1 \mathrm{MHz}$, PRF $10 \mathrm{~Hz}$, pulse duration $50 \mathrm{~ms}$, and total US stimulation period of $30 \mathrm{~s}$ ). As 
expected, for hMSCs without MBs and ATC stimulation (-US -MB), a small cell spread area of $30 \times 30 \mu \mathrm{m}^{2}$ led to reduced nuclear YAP in hMSCs $(22.30 \% \pm 3.81 \% ; n=3)$ compared with a large cell area of $100 \times 100 \mu \mathrm{m}^{2}(93.00 \% \pm 1.41 \% ; n=3)$ (Fig. 6C\&D). Importantly, ATC application rescued nuclear YAP in hMSCs pre-coated with MBs and with a small cell area of 30 $\times 30 \mu \mathrm{m}^{2}(41.93 \% \pm 2.78 \% ; n=3)($ Fig. $6 \mathrm{C} \& \mathrm{D})$. Together, these data support that ATC stimulation rescues YAP activity in hMSCs on soft substrates or with small cell spread areas. Since YAP is directly involved in the transcriptional program driving osteogenic differentiation of hMSCs (Fig. 5), our results strongly support that ATC stimulation rescues osteogenic differentiation of hMSCs on the soft PMA by promoting YAP nuclear localization.

\section{Actin, myosin II, and RhoA/ROCK signaling are all required for ATC-mediated YAP activation}

Actin filaments, in association with myosin II molecules, play a major role in cytoskeletal contractile forces. RhoA/ROCK signaling is also critically involved in regulating actin filament organization and cytoskeletal contractility $[5,14,15]$. We conducted experiments to examine the specific roles of actin, myosin II, and RhoA/ROCK signaling in regulating mechanoresponsive YAP activity in hMSCs subjected to ATC stimulation. hMSCs grown on the soft PMA were treated with small molecule drugs for $1 \mathrm{hr}$ before ATC stimulation to specifically inhibit actin polymerization (Cytochalasin D, $40 \mu \mathrm{M}$ ), myosin II activity (Blebbistatin, $50 \mu \mathrm{M}$ ), and RhoA/ROCK signaling (Y-27632, $50 \mu \mathrm{M})$. hMSCs were stained for YAP 30 min after ATC application (US conditions: acoustic pressure $0.08 \mathrm{MPa}$, center frequency $1 \mathrm{MHz}, \mathrm{PRF} 10 \mathrm{~Hz}$, pulse duration $50 \mathrm{~ms}$, and total US stimulation period of $30 \mathrm{~s}$ ). For hMSCs seeded on the soft PMA without MBs and ATC stimulation (-US -MB), treatments with Cytochalasin D, 
Blebbistatin, and Y-27632 led to significantly decreased nuclear YAP from $15.81 \% \pm 2.37 \%$ (for untreated control) to $6.79 \% \pm 1.26 \%$ (Cytochalasin $\mathrm{D} ; n=3), 7.80 \% \pm 1.76 \%$ (Blebbistatin; $n=$ 3), and $8.84 \% \pm 1.85 \%(\mathrm{Y}-27632 ; n=3)$, respectively (Fig. 7A\&B). Importantly, even though for untreated hMSCs coated with MBs, ATC stimulation led to significant increased nuclear YAP, treatments of hMSCs with Cytochalasin D, Blebbistatin, and Y-27632 completely inhibited mechanoresponsive YAP activity in hMSCs in response to ATC stimulation (Fig. 7). Together, our data support that actin, myosin II, and RhoA/ROCK signaling are all required for ATC-mediated YAP activation in hMSCs. Our results further support the notion that Rho GTPases, cytoskeletal contractility, and F-actin mechanics function upstream of mechanosensitive YAP signaling in controlling hMSC osteogenesis.

\section{CONCLUSION}

Mechano-sensitivity and -responsiveness to extracellular mechanical signals is a fundamental property that controls the function and behaviors of many types of mammalian cells, including hMSCs[19]. In this work, we have leveraged a novel ultrasound-based method, the ATC, to utilize US pulses to actuate functionalized lipid MBs covalently attached to the cell surface of hMSCs to exert subcellular mechanical forces to the cells $[25,26]$. US excitation of MBs elicits a rapid and sustained reactive cytoskeletal contractile force increase in mechanosensitive hMSCs $[25,26]$. In this work, we have further extended the application of ATC and unequivocally demonstrated its capability for improving hMSC osteogenesis under culture conditions that are normally repressive for hMSC osteogenesis (e.g., soft 2D cell culture surfaces or confined cell spread area). Since US excitation of MBs can be used for intracellular drug delivery (i.e., sonoporation)[36], we envision that sonoporation can be integrated into the same platform with 
ATC to deliver membrane-impermeable chemical components into hMSCs for additional modulations of intracellular signaling to further enhance hMSC osteogenesis. Since US application can be spatially and temporally controlled to a wide range of cells in both 2D and 3D environments, ATC can potentially provide a powerful technology for improving hMSC osteogenesis in both 2D and 3D culture environments. It remains an important future goal to determine whether ATC can promote osteogenesis of hMSCs embedded in 3D hydrogels and scaffolds commonly used for bone tissue constructs. A potential challenge is to control and maintain covalent attachments of functionalized MBs on the cell surface of hMSCs in 3D hydrogels and scaffolds before US excitation of MBs.

Physical factors in the local cellular microenvironment, including matrix mechanics, cell shape, and external mechanical forces, can all have strong influences on regulating hMSC differentiation[19]. Previous studies have also shown that YAP, as a transcription coactivator directly involved in the transcriptional program mediating hMSC osteogenesis, serves as the nuclear transducer in mediating force-sensitive hMSC osteogenesis [8, 9, 23, 24, 33]. Our data in this work support that hMSCs sense and respond to ATC stimulation through the force transmission across the mechanical continuum of MB-integrin-cytoskeleton, which further regulate downstream intracellular signaling to modulate intracellular cytoskeletal contractility. Importantly, ATC, by applying subcellular external forces to cells via integrin-anchored MBs, effectively increase cytoskeletal contractile force of hMSCs regardless of cell spread areas (Fig. 2). Interestingly, ATC stimulation rescues YAP activity (nuclear YAP) in hMSCs cultured on soft substrates and confined to small adhesive areas (Fig. 6), leading to enhanced hMSC osteogenesis, as evidenced by elevated ALP activity and expression levels of OCN, OPN, and BSP. Inhibition of cytoskeletal contractility by pharmaceutical drugs Cytochalasin D, 
Blebbistatin, and Y-27632 eliminates ATC-stimulated YAP activity, supporting the notion that actin, myosin II, and RhoA/ROCK signaling are all required for ATC-mediated YAP activation and they all function upstream of mechanosensitive YAP signaling in controlling mechanosensitive hMSC osteogenesis (Fig. 7). Altogether, these results demonstrate that mechanical stimulation via integrin-anchored MBs by ATC induces YAP activity in hMSCs by enhancing intracellular cytoskeletal contractility. In ATC-induced mechanotransduction in hMSCs, YAP functions as a downstream nuclear transducer and effector of cytoskeletal contractile force to mediate mechanosensitive hMSC osteogenesis.

In summary, we demonstrate for the first time that US excitation of MBs in the ATC could enable controlled application of subcellular mechanical stimuli to hMSCs to rescue hMSC osteogenesis in culture conditions that are intrinsically repressive for hMSC osteogenesis. ATC application activates transcriptional regulator YAP by enhancing cytoskeletal contractility to promote hMSC osteogenesis. Our data further show that F-actin, myosin II, and RhoA/ROCK signaling function upstream of YAP activity in mediating ATC-stimulated hMSC osteogenesis. We envision that ATC employing MBs as a biocompatible and multifunctional agent can be further developed in the future as an enabling cell mechanics and mechanobiology tool to regulate cell function, gene expression, and stem cell differentiation both in vitro and in vivo.

\section{ACKNOWLEDGEMENT}

This work is supported by the National Institutes of Health (R01 EB019436 and R21 EB017078 to J. F. and C. X. D.) and the National Science Foundation (CBET 1149401 to J. F.). The Lurie Nanofabrication Facility at the University of Michigan, a member of the National 
Nanotechnology Infrastructure Network (NNIN) funded by the National Science Foundation, is acknowledged for support in microfabrication. 


\section{REFERENCES}

[1] Wei X, Yang X, Han Z-p, Qu F-f, Shao L, Shi Y-f. Mesenchymal stem cells: a new trend for cell therapy. Acta Pharmacologica Sinica. 2013;34:747-54.

[2] Petite H, Viateau V, Bensaid W, Meunier A, de Pollak C, Bourguignon M, et al. Tissueengineered bone regeneration. Nature biotechnology. 2000;18:959-63.

[3] Tour G, Wendel M, Tcacencu I. Bone marrow stromal cells enhance the osteogenic properties of hydroxyapatite scaffolds by modulating the foreign body reaction. Journal of tissue engineering and regenerative medicine. 2014;8:841-9.

[4] Parekkadan B, Milwid JM. Mesenchymal stem cells as therapeutics. Annual review of biomedical engineering. 2010;12:87.

[5] McBeath R, Pirone DM, Nelson CM, Bhadriraju K, Chen CS. Cell shape, cytoskeletal tension, and RhoA regulate stem cell lineage commitment. Developmental cell. 2004;6:483-95.

[6] Kilian KA, Bugarija B, Lahn BT, Mrksich M. Geometric cues for directing the differentiation of mesenchymal stem cells. Proceedings of the National Academy of Sciences. 2010;107:4872-7. [7] Engler AJ, Sen S, Sweeney HL, Discher DE. Matrix elasticity directs stem cell lineage specification. Cell. 2006;126:677-89.

[8] Zhong W, Tian K, Zheng X, Li L, Zhang W, Wang S, et al. Mesenchymal stem cell and chondrocyte fates in a multishear microdevice are regulated by Yes-associated protein. Stem cells and development. 2013;22:2083-93.

[9] Kim KM, Choi YJ, Hwang J-H, Kim AR, Cho HJ, Hwang ES, et al. Shear stress induced by an interstitial level of slow flow increases the osteogenic differentiation of mesenchymal stem cells through TAZ activation. PloS one. 2014;9:e92427. 
[10] Steward AJ, Kelly DJ. Mechanical regulation of mesenchymal stem cell differentiation. Journal of anatomy. 2015;227:717-31.

[11] Sonam S, Sathe SR, Yim EK, Sheetz MP, Lim CT. Cell contractility arising from topography and shear flow determines human mesenchymal stem cell fate. Scientific reports. $2016 ; 6$.

[12] Huebsch N, Arany PR, Mao AS, Shvartsman D, Ali OA, Bencherif SA, et al. Harnessing traction-mediated manipulation of the cell/matrix interface to control stem-cell fate. Nature materials. 2010;9:518-26.

[13] Fu J, Wang Y-K, Yang MT, Desai RA, Yu X, Liu Z, et al. Mechanical regulation of cell function with geometrically modulated elastomeric substrates. Nature methods. 2010;7:733-6. [14] Wang Y-K, Yu X, Cohen DM, Wozniak MA, Yang MT, Gao L, et al. Bone morphogenetic protein-2-induced signaling and osteogenesis is regulated by cell shape, RhoA/ROCK, and cytoskeletal tension. Stem cells and development. 2011;21:1176-86.

[15] Khatiwala CB, Kim PD, Peyton SR, Putnam AJ. ECM compliance regulates osteogenesis by influencing MAPK signaling downstream of RhoA and ROCK. Journal of Bone and Mineral Research. 2009;24:886-98.

[16] Tee S-Y, Fu J, Chen CS, Janmey PA. Cell shape and substrate rigidity both regulate cell stiffness. Biophysical journal. 2011;100:L25-L7.

[17] Teo BKK, Wong ST, Lim CK, Kung TY, Yap CH, Ramagopal Y, et al. Nanotopography modulates mechanotransduction of stem cells and induces differentiation through focal adhesion kinase. Acs Nano. 2013;7:4785-98.

[18] Rape AD, Guo W-h, Wang Y-l. The regulation of traction force in relation to cell shape and focal adhesions. Biomaterials. 2011;32:2043-51. 
[19] Sun Y, Chen CS, Fu J. Forcing stem cells to behave: a biophysical perspective of the cellular microenvironment. Annual review of biophysics. 2012;41:519-42.

[20] Sun Y, Shao Y, Xue X, Fu J. Emerging Roles of YAP/TAZ in Mechanobiology. Molecular and Cellular Mechanobiology: Springer; 2016. p. 83-96.

[21] Zhao B, Tumaneng K, Guan K-L. The Hippo pathway in organ size control, tissue regeneration and stem cell self-renewal. Nature cell biology. 2011;13:877-83.

[22] Halder G, Johnson RL. Hippo signaling: growth control and beyond. Development. 2011;138:9-22.

[23] Dupont S, Morsut L, Aragona M, Enzo E, Giulitti S, Cordenonsi M, et al. Role of YAP/TAZ in mechanotransduction. Nature. 2011;474:179-83.

[24] Hong J-H, Hwang ES, McManus MT, Amsterdam A, Tian Y, Kalmukova R, et al. TAZ, a transcriptional modulator of mesenchymal stem cell differentiation. Science. 2005;309:1074-8. [25] Fan Z, Sun Y, Chen D, Tay D, Chen W, Deng CX, et al. Acoustic tweezing cytometry for live-cell subcellular modulation of intracellular cytoskeleton contractility. Scientific reports. $2013 ; 3$.

[26] Chen D, Sun Y, Gudur MS, Hsiao Y-S, Wu Z, Fu J, et al. Two-Bubble Acoustic Tweezing Cytometry for Biomechanical Probing and Stimulation of Cells. Biophysical journal. 2015;108:32-42.

[27] Chen D, Sun Y, Deng CX, Fu J. Improving survival of disassociated human embryonic stem cells by mechanical stimulation using acoustic tweezing cytometry. Biophysical journal. 2015;108:1315-7. 
[28] Yang MT, Fu J, Wang Y-K, Desai RA, Chen CS. Assaying stem cell mechanobiology on microfabricated elastomeric substrates with geometrically modulated rigidity. Nature protocols. 2011;6:187-213.

[29] Saez A, Buguin A, Silberzan P, Ladoux B. Is the mechanical activity of epithelial cells controlled by deformations or forces? Biophysical journal. 2005;89:L52-L4.

[30] Tan JL, Tien J, Pirone DM, Gray DS, Bhadriraju K, Chen CS. Cells lying on a bed of microneedles: an approach to isolate mechanical force. Proceedings of the National Academy of Sciences. 2003;100:1484-9.

[31] Webster KD, Ng WP, Fletcher DA. Tensional homeostasis in single fibroblasts. Biophysical journal. 2014;107:146-55.

[32] Weng S, Shao Y, Chen W, Fu J. Mechanosensitive subcellular rheostasis drives emergent single-cell mechanical homeostasis. Nature materials. 2016.

[33] Halder G, Dupont S, Piccolo S. Transduction of mechanical and cytoskeletal cues by YAP and TAZ. Nature reviews Molecular cell biology. 2012;13:591-600.

[34] Sun Y, Yong KMA, Villa-Diaz LG, Zhang X, Chen W, Philson R, et al. Hippo/YAPmediated rigidity-dependent motor neuron differentiation of human pluripotent stem cells. Nature materials. 2014;13:599-604.

[35] Wang C, Zhu X, Feng W, Yu Y, Jeong K, Guo W, et al. Verteporfin inhibits YAP function through up-regulating 14-3-3 $\sigma$ sequestering YAP in the cytoplasm. American journal of cancer research. 2016;6:27.

[36] Fan Z, Liu H, Mayer M, Deng CX. Spatiotemporally controlled single cell sonoporation. Proceedings of the National Academy of Sciences. 2012;109:16486-91. 


\section{FIGURES AND FIGURE CAPTIONS}
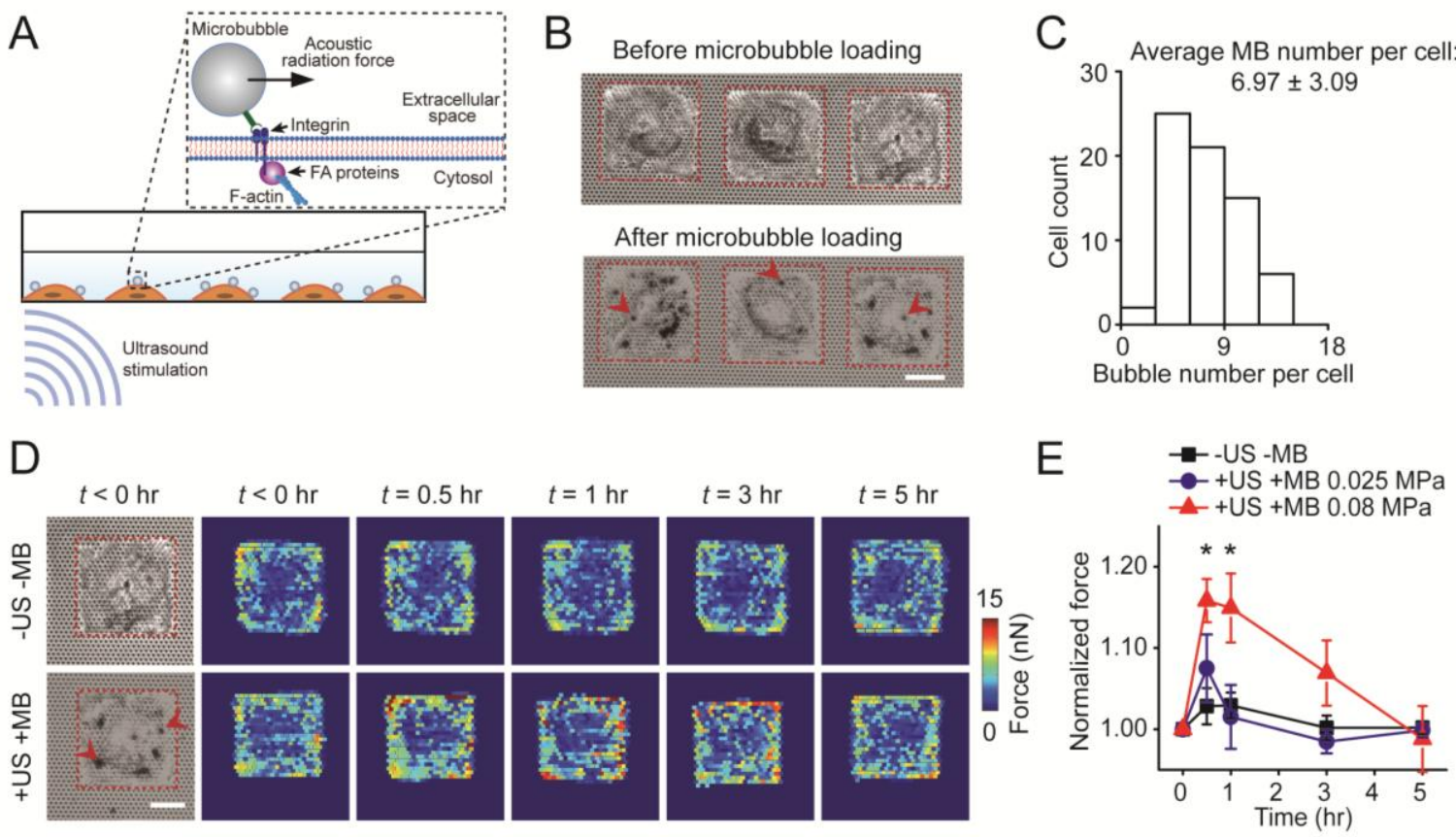

Figure 1. Acoustic tweezing cytometry (ATC) modulates cytoskeletal contractility of human mesenchymal stem cells (hMSCs). (A) Schematic showing ultrasound (US) excitation of functionalized microbubbles (MBs) attached to a cell via RGD-integrin binding. US excitation of MBs led to an acoustic radiation force acting on the MBs, resulting in lateral displacement of MBs and thus mechanical stimulation of the MB-integrin-F-actin linkage. (B) Bright field images showing micropatterned single hMSCs seeded on the PDMS micropost array (PMA) before and after loading MBs on hMSCs. Arrow heads mark representative MBs. Red rectangles in micrographs highlight cell boundaries. Scale bar, $30 \mu \mathrm{m}$. (C) Distribution of MB number per cell. The average MB number per cell was $6.97 \pm 3.09$ (mean \pm standard deviation). The MB number per cell was counted based on bright field images shown in Fig. 1B. (D) Subcellular contractile force distribution for micropatterned single hMSCs loaded with MBs before $(t<0 \mathrm{hr})$ and after $(t>0$ hr) US stimulation (+US +MB; bottom). Dynamic evolution of subcellular contractile forces of single hMSCs without MBs and US stimulation (-US - MB; top) was also 
shown for comparison. Scale bar, $30 \mu \mathrm{m}$. (E) Change in total cytoskeletal contractile force of micropatterned single hMSCs (relative to pre-US application values) as a function of time. Error bar denotes SEM, with $n=7$ for acoustic pressure of $0.025 \mathrm{MPa}$ and $n=6$ for acoustic pressure of $0.08 \mathrm{MPa}$. Control group without MBs and US stimulation (-US -MB, $n=7$ ) was included for comparison. $P$-values were calculated using two group $t$-test with respect to control. $*, P<0.05$. The post height of the PMA used for cytoskeletal contractility measurements was $7.1 \mu \mathrm{m}$. US parameters: frequency $1 \mathrm{MHz}$, pulse repetition frequency $10 \mathrm{~Hz}$, pulse duration $50 \mathrm{~ms}$, treatment duration $30 \mathrm{~s}$, and acoustic pressure $0.08 \mathrm{MPa}$ or as indicated. 


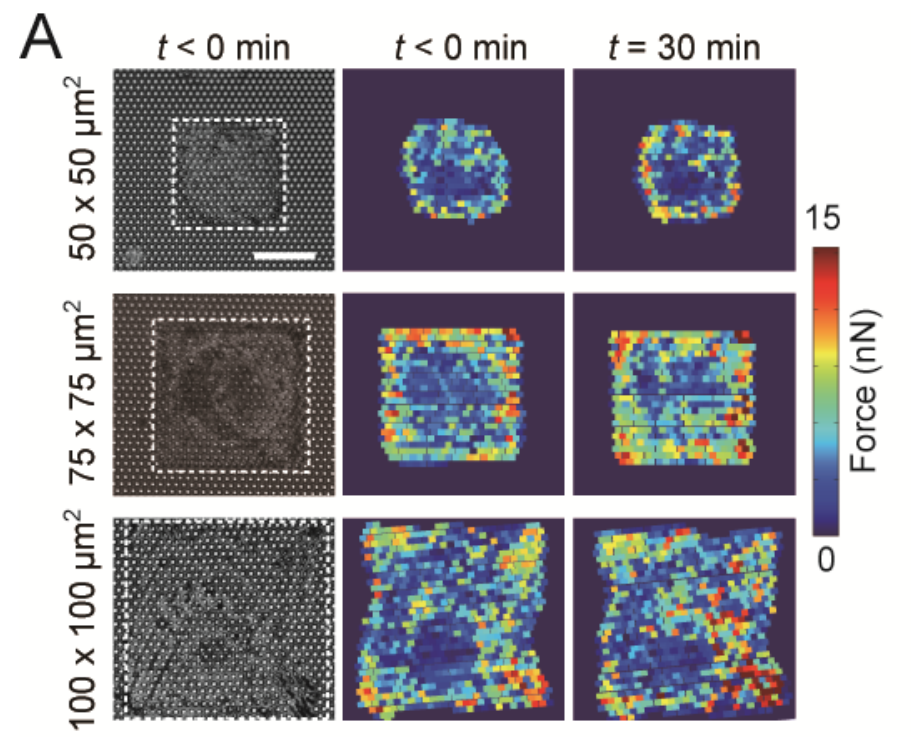

B
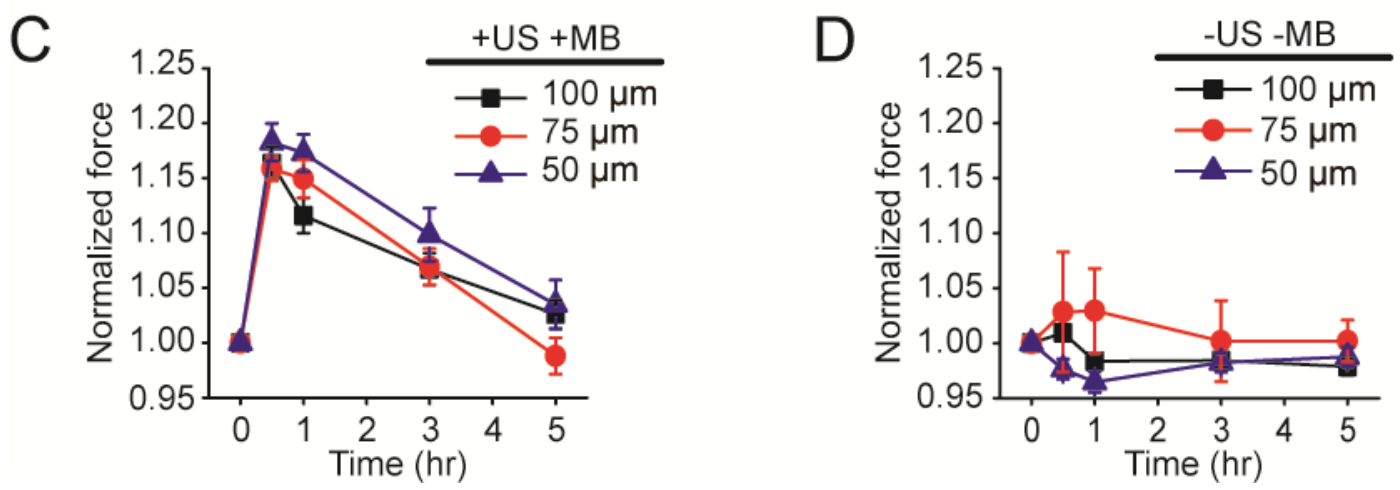

Figure 2. ATC is effective in modulating cytoskeletal contractile force of hMSCs with different cell areas. (A) Bright field images showing micropatterned single hMSCs on the PDMS micropost array (PMA) with different cell areas as indicated. Subcellular cytoskeletal contractile force distributions before $(t<0 \mathrm{~min}$ ) and $30 \mathrm{~min}$ after ultrasound (US) stimulation were shown for the same single cells. White rectangles in micrographs highlight cell boundaries. Scale bar, $30 \mu \mathrm{m}$. (B) Total cytoskeletal contractile force of micropatterned single hMSCs before US stimulation as a function of cell area. A linear function (black line) was used for fitting, $R^{2}=$ 0.9817. (C\&D) Dynamic evolution of total cytoskeletal contractile force of single hMSCs with different cell areas (normalized to pre-US application values). C, cells were loaded with MBs before being stimulated with US (+ US +MB). D, control group without MBs and US stimulation 
(-US -MB). Error bar denotes SEM, with $n=6$ - 10. US parameters: frequency $1 \mathrm{MHz}$, pulse repetition frequency $10 \mathrm{~Hz}$, pulse duration $50 \mathrm{~ms}$, treatment duration $30 \mathrm{~s}$, and acoustic pressure $0.08 \mathrm{MPa}$. 
A

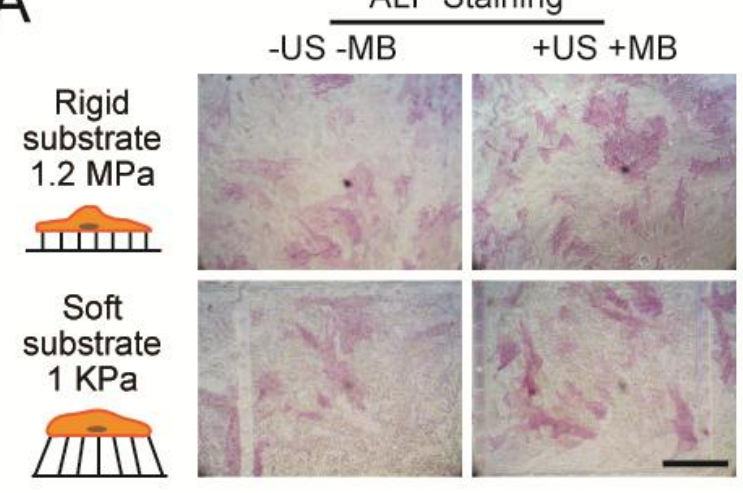

B

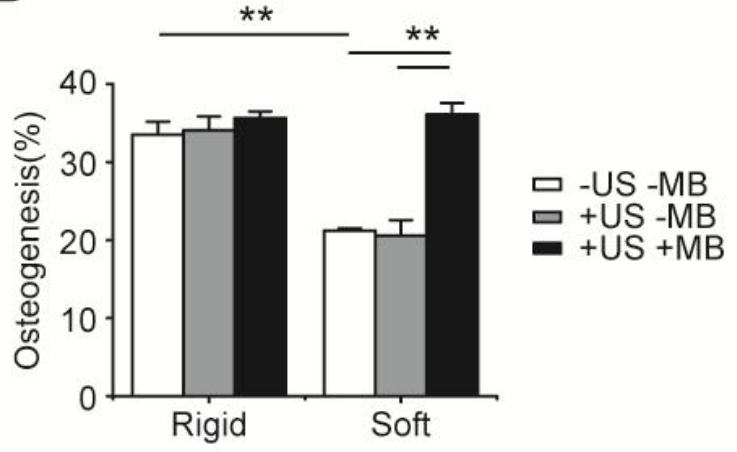

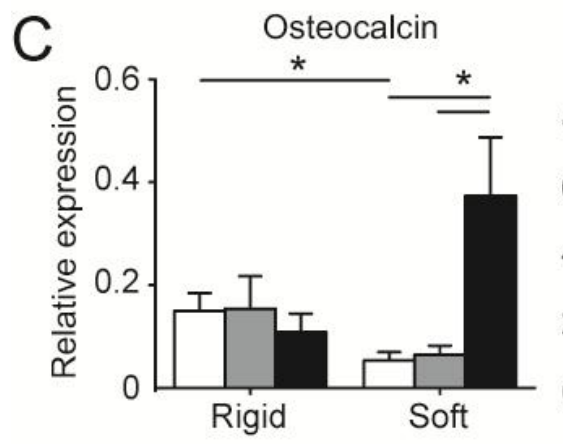
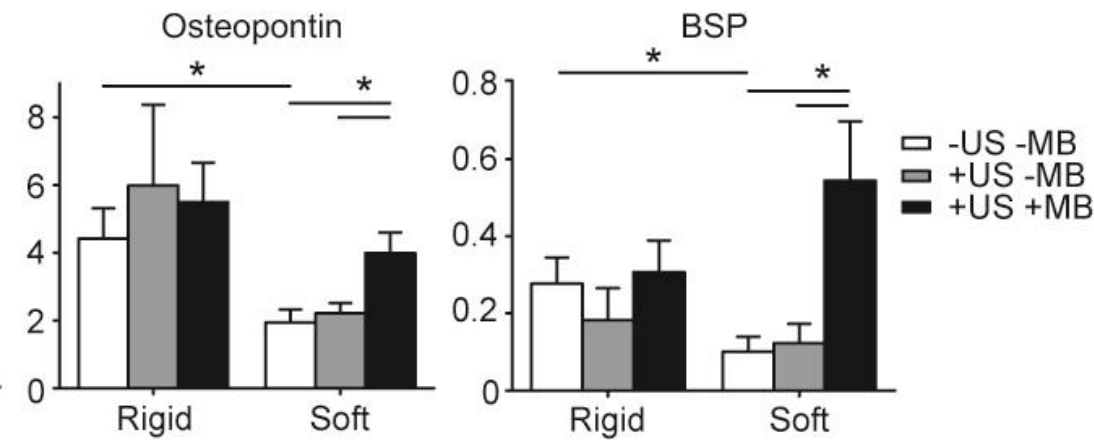

Figure 3. ATC rescues osteogenic differentiation of hMSCs on soft substrates. (A) Bright field images showing hMSCs stained for ALP after $6 \mathrm{~d}$ of culture in osteogenic differentiation medium on PDMS micropost arrays (PMAs) with different rigidities as indicated. hMSCs were loaded with microbubbles (MBs) before ultrasound (US) stimulation (+US +MB). Negative control of hMSCs without MBs and US stimulation (-US -MB) was included for comparison. Scale bar, $250 \mu \mathrm{m}$. (B) Bar plot showing percentage of hMSC osteogenesis as a function of substrate rigidity under different conditions as indicated. hMSCs without MBs but stimulated with US (+US -MB) were included for comparison. (C) Relative expression of osteogenic markers in hMSCs cultured on PMAs with different rigidities. Expression levels were normalized to the value of TBP gene. For +US +MB group, hMSCs were stimulated using US each day for $5 \mathrm{~min}$, with US parameters being frequency $1 \mathrm{MHz}$, pulse repetition frequency 10 $\mathrm{Hz}$, pulse duration $50 \mathrm{~ms}$, treatment duration $5 \mathrm{~min}$, and acoustic pressure $0.08 \mathrm{MPa}$. Each day before US stimulation, cells were re-coated with MBs with the average MB number per cell kept 
as constant. Error bar denotes SEM, with $n \geq 3$. *,$P<0.05$; **, $P<0.01$. 


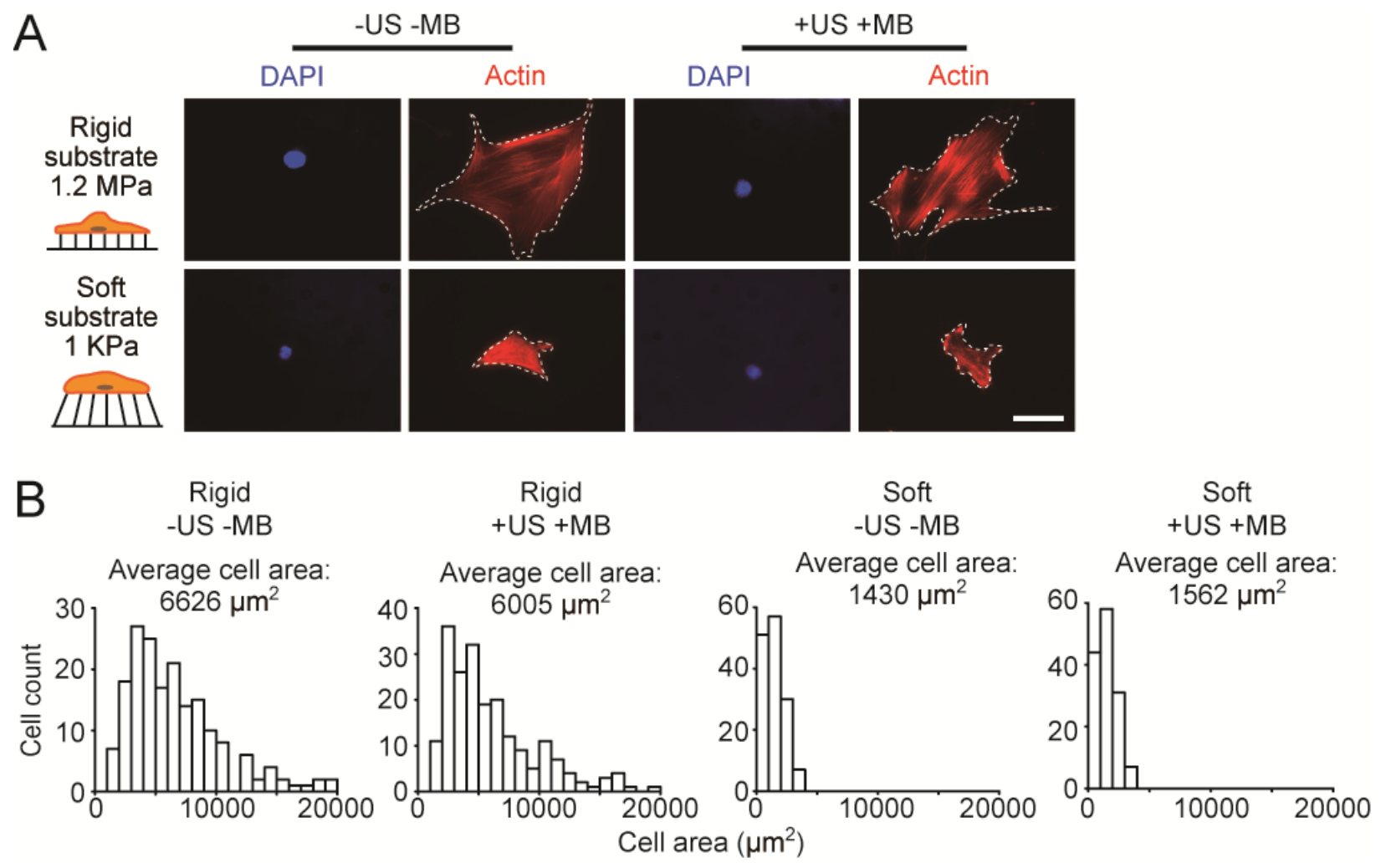

Figure 4. ATC does not affect cell spreading of hMSCs on rigid or soft substrates. (A)

Representative immunofluorescence images showing single hMSCs seeded on the PDMS micropost arrays (PMAs) with different rigidities as indicated. hMSCs were loaded with microbubbles (MBs) before ultrasound (US) stimulation (+US +MB). Negative control for hMSCs without MBs and US stimulation (-US -MB) was included for comparison. Cells were stained with phalloidin (red) and DAPI (blue) 30 min after US stimulation. White dashed lines in micrographs highlight cell boundaries. Scale bar, $50 \mu \mathrm{m}$. (B) Histograms showing distributions of cell area of hMSCs plated on PMAs with different rigidities under -US -MB or +US +MB conditions as indicated. US parameter: frequency $1 \mathrm{MHz}$, pulse repetition frequency $10 \mathrm{~Hz}$, pulse duration $50 \mathrm{~ms}$, treatment duration $5 \mathrm{~min}$, and acoustic pressure $0.08 \mathrm{MPa}$. 
A

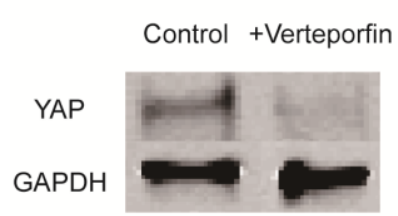

B

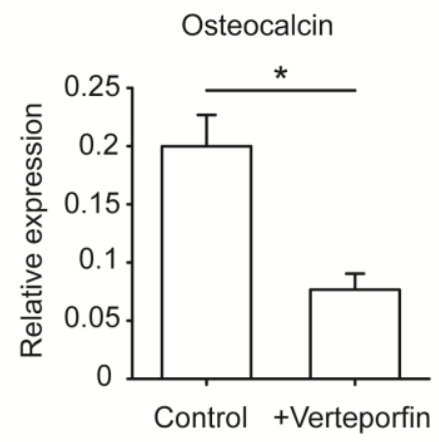

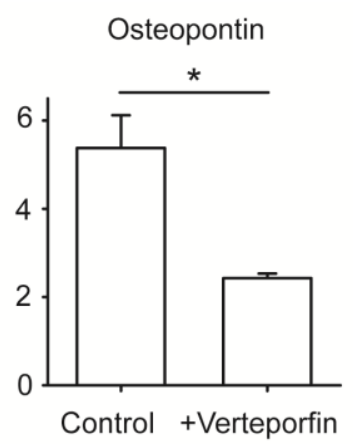

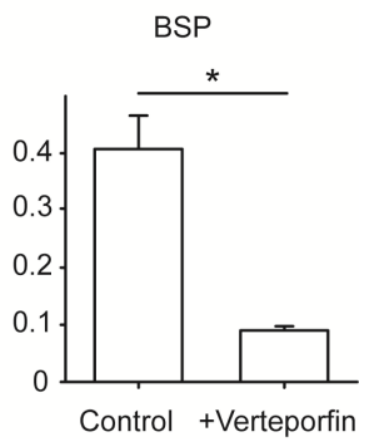

Figure 5. YAP activity drives osteogenic differentiation of hMSCs. (A) Western blot showing YAP expression level in hMSCs treated with or without YAP inhibitor verteporfin $(5 \mu \mathrm{M})$ for 2 $\mathrm{d}$ as indicated. GAPDH was used as a loading control. (B) Relative expression of osteogenic markers in hMSCs after $6 \mathrm{~d}$ of culture in osteogenic differentiation medium supplemented with or without verteporfin $(5 \mu \mathrm{M})$. Error bar denotes SEM, with $n \geq 3$. ${ }^{*}, P<0.05$. 

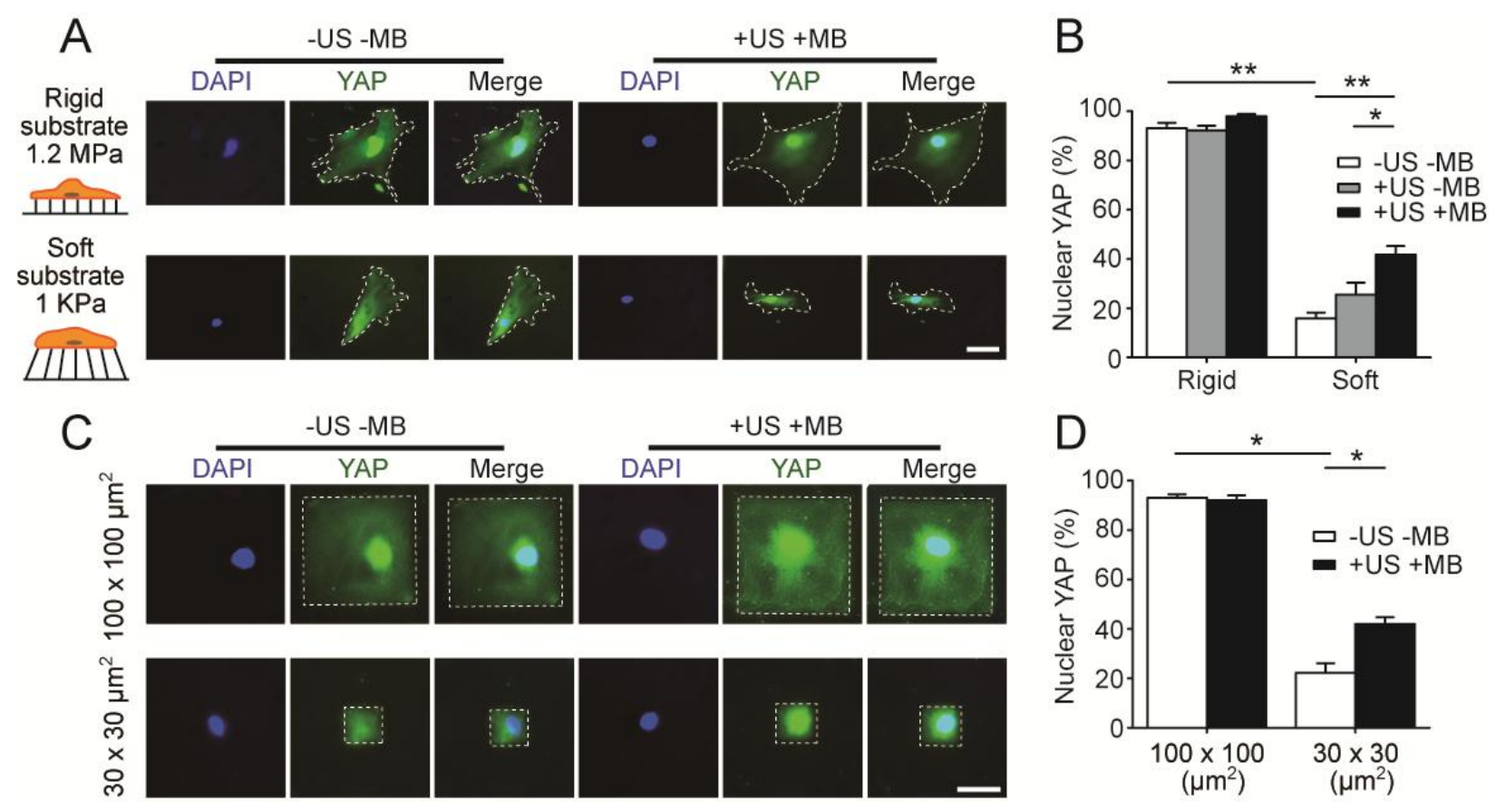

Figure 6. ATC rescues YAP activity in hMSCs seeded on soft substrates or with small cell areas. (A\&B) Representative fluorescence images (A) and bar plot (B) showing subcellular localization of YAP in hMSCs on the PDMS micropost arrays (PMAs) with different rigidities as indicated. hMSCs were loaded with functionalized microbubbles (MBs) before ultrasound (US) stimulation (+US +MB). Negative control of hMSCs without MBs and US stimulation (-US -MB) was included for comparison. White dashed lines in micrographs highlight cell boundaries. Scale bar in $A, 50 \mu \mathrm{m}$. (C\&D) Representative fluorescence images (C) and bar plot (D) showing subcellular YAP localization in micropatterned hMSCs with different cell areas as indicated. White rectangles in micrographs highlight cell boundaries. Scale bar in C, $50 \mu \mathrm{m}$. hMSCs were fixed and stained for YAP 30 min after US stimulation. Error bar denotes SEM. with $n \geq 3 .{ }^{*}, P$ $<0.05$;**, $P<0.01$. US parameter: frequency $1 \mathrm{MHz}$, pulse repetition frequency $10 \mathrm{~Hz}$, pulse duration $50 \mathrm{~ms}$, treatment duration $5 \mathrm{~min}$, and acoustic pressure $0.08 \mathrm{MPa}$. 


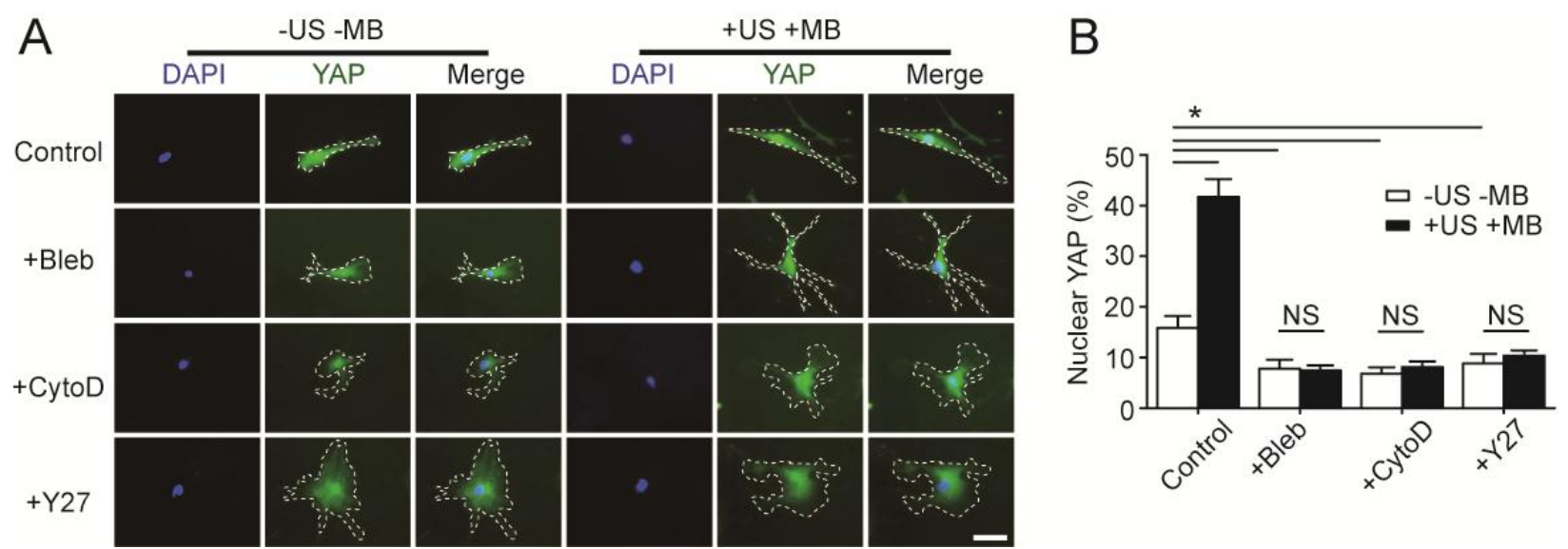

Figure 7. Actin, myosin II, and RhoA/ROCK signaling are all required for rescuing YAP activity in hMSCs on soft substrates by ATC. (A\&B) Representative fluorescence images (A) and bar plot (B) showing subcellular YAP localization in hMSCs seeded on a soft PMA (effective substrate rigidity $E_{\text {eff }}=1 \mathrm{kPa}$ ) under different drug treatments as indicated. hMSCs were coated with functionalized microbubbles (MBs) before ultrasound (US) stimulation (+US $+\mathrm{MB}$ ). Negative control for hMSCs without MBs and US stimulation (-US -MB) was included for comparison. Cells were treated with pharmacological drugs for $1 \mathrm{hr}$ before US application. Blebbistatin, or Bleb, $50 \mu \mathrm{M}$; Cytochalasin D, or CytoD, $40 \mu \mathrm{M}$; Y-27632, or Y27, $50 \mu \mathrm{M}$. Cells were stained for YAP 30 min after US stimulation. White dashed lines in micrographs highlight cell boundaries. Scale bar, $50 \mu \mathrm{m}$. Error bar, the mean \pm s.e.m, with $n \geq 3 .{ }^{*}, P<0.05$; NS, $P>$ 0.05. US parameter: frequency $1 \mathrm{MHz}$, pulse repetition frequency $10 \mathrm{~Hz}$, pulse duration $50 \mathrm{~ms}$, treatment duration $5 \mathrm{~min}$, and acoustic pressure $0.08 \mathrm{MPa}$. 